\title{
Anders Hanson: Workplace Health Promotion: A Salutogenic Approach
}

\author{
AuthorHouse, Bloomington, 2007, 351 pages. ISBN: 978-1-4259-9726-7, $19.99 €$, \$22.00
}

\author{
Natalie Schmitt
}

Published online: 14 November 2008

(C) Springer-Verlag 2008

Workplace Health Promotion is the English-language version of the original Swedish edition HälsopromotionIArbetslivet which appeared in 2004 by the occupational and organisational psychologist Anders Hanson.

Hanson devotes the first of the 12 chapters to the analysis of the present working life conditions, strategies in health work, the two-dimensional model of health including salutogenesis and pathogenesis, a historical overview of (workplace) health promotion, the significance of the existence of theories, and the various disciplines involved in workplace health promotion. The author claims domination of social and behavioural sciences in the field of workplace health promotion and chose to build his overarching framework for health promotion at the workplace on Antonovsky's concept of sense of coherence which is described in depth in Chap. 6. Chapters 8-12 of the book deal with the salutogenic perspective of health promotion and its four criteria which form the basis of health promotion work: focus on measures promoting health, thinking in terms of setting, participation, and process. There is a summary at the end of each chapter and a bibliography with 120 entries at the end of the book, of which about $50 \%$ are Swedish. An index is missing, unfortunately.
The book is mostly theoretical in character, but it also elucidates practical issues. However, the latter focus on Swedish culture and workplace conditions, although the author derived a lot of knowledge and inspiration from WHO and the European Network for Workplace Health Promotion (ENWHP). Gender-specific aspects of workplace health promotion, which are not approached in the book, may not be essential in Sweden but in other countries of the world, for example.

Workplace Health Promotion should thus not only arouse interest of both employers and employees, it is also suitable as a course book for students of public health, health promotion, occupational health and preventive medicine. The writing style and presentation of the book are attractive to all different kinds of interested readers. Hanson succeeded in filling the gap in publications which are scientifically based and precise on the one hand and provide realistic solutions and practical approaches to reduce absenteeism from work due to illness on the other hand. His book includes both indepth descriptions of general principles and effective strategies essential to practitioners in the field of workplace health promotion.

\footnotetext{
N. Schmitt $(\bowtie)$

Research Association Public Health, Faculty of Medicine,

Technische Universität Dresden,

Fiedlerstr. 33,

01307 Dresden, Germany

e-mail: Natalie.Schmitt@tu-dresden.de
} 